\title{
Evaluación a través de tomografía computarizada del efecto de una infusión endovenosa de ketamina en el desarrollo de atelectasia inducida por anestesia general en perros
}

\author{
Evaluation of an endovenous ketamine infusion through computed tomography \\ on the development of pulmonary atelectasis due to general anesthesia in dogs \\ CA Henríquez ${ }^{\mathrm{a}}$, LM Mieres ${ }^{{ }^{*}}$, HA Bustamante ${ }^{\mathrm{b}}$, DE Herzberg $^{\mathrm{b}}$, CE Campillo ${ }^{\mathrm{a}}$, MP Cabrera ${ }^{\mathrm{b}}$, MA Gómez ${ }^{\mathrm{c}}$ \\ aPrograma de Doctorado en Ciencias Veterinarias, Universidad Austral de Chile, Valdivia, Chile. \\ ${ }^{b}$ Instituto de Medicina Preventiva Veterinaria, Universidad Austral de Chile, Valdivia, Chile. \\ 'Instituto de Farmacología y Morfofisiología, Universidad Austral de Chile, Valdivia, Chile.
}

\begin{abstract}
SUMMARY
The aim of this study was to assess through computed tomography the presence of pulmonary atelectasis in dogs under inhalatory anesthesia and evaluate the effect of an endovenous ketamine infusion upon it. For this purpose 12 dogs separated in two groups (A and B) of 6 dogs each were used. Both groups were subjected to the same anesthetic protocol. The protocol consisted in premedication with xilacine IM, induction with propofol IV and maintenance with inhalatory anesthesia for a period of two hours. The group B received also a ketamine infusion. Computed tomographic images were taken at 0,60 and 120 minutes, with the purpose of monitoring developments of atelectasis. In 58\% of the dogs it was possible to determine the presence of some degree of atelectasis. In these animals atelectasis was observed in all the cases, but only in one lung. Atelectasis was found in 5 animals in the group without infusion of ketamine and in 2 animals in the group with infusion. The collapsed lung zones ranged between 0.13 and $8.03 \mathrm{~cm}^{2}$ in group $\mathrm{A}$, and 0.07 and $2.10 \mathrm{~cm}^{2}$ in group B. Results indicated that ketamina infusion did not influence the presentation of pulmonary atelectasis. With regard to the evolution of the atelectasis through time, slight changes were observed in both groups, not being statistically significant $(\mathrm{P}>0.05)$.
\end{abstract}

Palabras clave: atelectasia, anestesia, tomografía computarizada, perro.

Key words: atelectasis, anesthesia, computed tomography, dog.

\section{INTRODUCCIÓN}

El intercambio gaseoso óptimo requiere el transporte conjunto de aire y de la sangre hasta el alvéolo (ventilación y flujo sanguíneo), por lo que la relación de ventilación y perfusión (V/Q) es vital. De forma ideal el pulmón debiera recibir volúmenes equilibrados tanto de ventilación como de perfusión. En condiciones reales, existe un cierto desequilibrio V/Q en parte como resultado de la acción de las fuerzas de gravedad sobre el pulmón, como se aprecia en animales de gran tamaño (como por ejemplo equinos) en decúbito, sobre todo supino y lateral (Robinson 2003).

$\mathrm{La}$ anestesia general y enfermedades pulmonares acentúan el desequilibrio V/Q y por lo tanto perjudican el intercambio de gases (Robinson 2003).

En humanos se puede encontrar aproximadamente entre 16 a $20 \%$ del parénquima pulmonar hipoventilado o colapsado durante la anestesia, generando zonas de baja relación V/Q y shunt pulmonar (Hedenstierna y col 1986). El colapso ocurre con regularidad durante el periodo de inducción anestésica, pero puede persistir aún después

Aceptado: 06.10.2010.

* Casilla N 567, Valdivia, Chile; mmieres@uach.cl de finalizado el proceso anestésico y contribuye significativamente a aumentar los gastos de atención de salud, la convalecencia e incluso aumenta la probabilidad de enfermedad y muerte (Magnusson y Spahn 2003).

En la década de los ochenta fue diagnosticado en humanos por primera vez el colapso alveolar producido por anestesia. Esto fue observado tanto en pacientes neonatos como adultos gracias a la introducción de nuevas técnicas de rayos $\mathrm{X}$ como la tomografía computarizada (TC) (Magnusson y Spahn 2003). Este colapso alveolar se denominó atelectasia pulmonar, la cual era producida por la anestesia (Tusman y col 2002). En 1985, Brismar y col mostraron que 5 minutos posterior a la inducción de la anestesia aparecían picos de densidad en ciertas áreas de ambos pulmones. Luego Hedenstierna y col (1989) también encontraron estos aumentos de densidad en ovejas anestesiadas, demostrando a través de microscopía que estas densidades se trataban de regiones pulmonares atelectásicas (Pedersen y col 1992). La cantidad de tejido atelectásico está determinada por la magnitud del shunt producido por la anestesia (Warner y col 1996).

La atelectasia pulmonar aparece en el $90 \%$ de los pacientes humanos sometidos a un procedimiento de anestesia general (Gunnarsson y col 1991). Esto convierte a la atelectasia en una de las complicaciones más frecuentes en pacientes quirúrgicos. Se puede presentar de forma 
subclínica y resolverse espontáneamente en 24 a 48 horas o persistir por mayor tiempo (Brooks-Brunn 1995).

En general se describen distintos factores que influyen en la presentación y severidad de la atelectasia pulmonar durante un proceso anestésico. Dentro de estos factores destaca el posicionamiento, siendo más severa cuando los pacientes mantienen un decúbito supino, presentándose de manera más pronunciada cercana al diafragma, disminuyendo hacia el ápex pulmonar (Reber y col 1996). Además se ve asociada con la edad, siendo mucho más severa en pacientes pediátricos. Condición corporal, Strandberg y col (1987), utilizando imágenes de tomografía, observaron una alta correlación entre el porcentaje de atelectasia y la masa corporal, explicando la mayor presentación de ésta en pacientes con obesidad. Otro factor es el porcentaje de oxígeno inspirado, siendo este punto en el que se ha puesto mayor atención en el último tiempo, ya que se ha descubierto que a una menor concentración de oxígeno inspirado el porcentaje de atelectasia presentado es menor y se presenta luego de un tiempo mayor de anestesia (Staffieri y col 2007). Por último se destaca el tipo de anestésico utilizado, ya que por ejemplo la ketamina no produce atelectasia.

La ketamina es un anestésico inyectable, que pertenece junto a la fenciclidina y la tiletamina al grupo de los anestésicos disociativos (Sumano y Ocampo 1997). En pacientes con ventilación espontánea la administración de la mayoría de los anestésicos causa una inmediata baja de la Capacidad Residual Funcional (CRF), lo cual resulta en un aumento de la diferencia entre la oxigenación arterial y alveolar (Gooding y col 1977). El uso de anestésicos que mantengan el tono muscular pulmonar previene la aparición de atelectasia. La ketamina aparece como el único anestésico con la capacidad de mantener la CRF y por ende prevenir la presentación de atelectasia, reduciendo así la capacidad de presentar hipoxemia intraoperatoria (Tweed y col 1972, Gooding y col 1977, Tokics y col 1987, Hedenstierna 2003).

Durante la anestesia con ketamina en pacientes con ventilación espontánea el volumen total puede ser mantenido al mismo nivel que en estado consciente (Tokics y col 1987). Además, hay cambios mínimos en el intercambio gaseoso y ausencia de atelectasia y shunt. Al parecer esto sería debido a la mantención del tono del músculo esquelético y a la mantención de la presión vascular (Hass y Harper 1992). Ketamina administrada en perros en infusiones de $10 \mathrm{ug} / \mathrm{kg} / \mathrm{min}$ otorga analgesia adecuada en pacientes sometidos a intervenciones quirúrgicas (Wagner y col 2002) y es capaz de reducir la concentración alveolar mínima de isoflurano en un 25\% (Muir y col 2003). Adicionalmente, en el estudio de Muir y col (2003), el efecto analgésico de ketamina se asoció a un incremento significativo de la presión arterial media (PAM) en comparación a la PAM de perros mantenidos anestésicamente sólo con isoflurano. Previamente otros autores han señalado el efecto analgésico de dosis bajas de ketamina en perros sin reportar efectos adversos asociados (Ko y col 2001, Slingsby y
Waterman-Pearson 2000), sin embargo, no se ha reportado si ketamina administrada en la dosis mencionada anteriormente puede reducir el porcentaje de atelectasia pulmonar en perros sometidos a anestesia general inhalatoria.

La TC ha emergido como el método imagenológico preferido para el pulmón, debido a la amplitud de niveles de atenuación entre las estructuras, gran resolución y velocidad. A partir de las imágenes pulmonares obtenidas mediante tomografía computarizada es posible medir el volumen, distribución y comportamiento de la ventilación bajo varias condiciones clínicas (Duggan y Kavanagh 2005).

En contexto de lo anterior se planteó como hipótesis que la administración de una infusión endovenosa de ketamina en perros bajo anestesia general inhalatoria previene el desarrollo de atelectasia pulmonar. Para ello se establecieron como objetivos: determinar el porcentaje de atelectasia pulmonar en caninos sometidos a anestesia inhalatoria y el efecto de una infusión endovenosa de ketamina sobre la presentación de atelectasia pulmonar en caninos bajo anestesia inhalatoria.

\section{MATERIAL Y MÉTODOS}

El estudio se realizó en el Hospital Clínico Veterinario de la Universidad Austral de Chile. Se utilizaron 12 caninos (Canis lupus familiaris), entre 2 y 6 años de edad, con un peso entre 25 y 45 kilogramos, sin distinción de sexo y raza, clínicamente sanos y con una condición corporal de 3 (escala de 1-5). Tanto el mantenimiento como el procedimiento realizado en ellos estuvieron de acuerdo con las directrices de la guía para el cuidado y uso de animales de laboratorio.

A los animales se les retiraron los alimentos sólidos y líquidos doce y seis horas, respectivamente, antes del inicio del estudio. Cada animal fue premedicado con xilacina a una dosis de $1 \mathrm{mg} / \mathrm{kg}$ por vía intramuscular. Posterior a la premedicación se realizó la inducción anestésica administrando propofol a una dosis de $4,4 \mathrm{mg} / \mathrm{kg}$. Luego los animales fueron intubados y la anestesia fue mantenida con isoflurano en $\mathrm{O}_{2}$ a una concentración continua durante todo el estudio ( $1 \%$ a $2 \%$ vol.).

Los animales fueron separados en dos grupos: un grupo "A", el cual está comprendido por seis animales a los cuales se les mantuvo únicamente bajo anestesia inhalatoria con isoflurano y un grupo "B", comprendido por los otros seis animales, el cual fue mantenido mediante anestesia inhalatoria con isoflurano, más la administración de una infusión endovenosa de $10 \mu \mathrm{g} / \mathrm{kg} / \mathrm{min}$ de ketamina (Muir y col 2003). Para esto $60 \mathrm{mg}$ de ketamina fueron diluidos en un litro de cristaloide ( $\mathrm{NaCl}$ 0,9\%), el cual se administró a una razón de $10 \mathrm{~mL} / \mathrm{kg} / \mathrm{h}$, utilizando un equipo de infusión BRAUN, Infusomat ${ }^{\circledR} \mathrm{fmS}$. Ambos grupos de animales se mantuvieron anestesiados durante un periodo de dos horas, tiempo durante el cual se monitorizaron sus frecuencias y constantes (frecuencia respiratoria, frecuencia cardiaca, presión arterial, saturación de oxígeno). 
Una vez anestesiados los animales, se procedió a la toma de dos radiografías por individuo, una en decúbito lateral derecho y otra en decúbito lateral izquierdo, con el fin de descartar la presencia de alguna enfermedad pulmonar preexistente.

Los animales fueron posicionados en la mesa de tomografía en decúbito prono con las cuatro extremidades en extensión. El examen tomográfico de la cavidad torácica fue realizado con un equipo TC de $4^{\text {a }}$ generación Picker JQ/ PQ 4,25 Diagnostic, utilizándose los parámetros técnicos de $130 \mathrm{kV}$ y $85 \mathrm{~mA}$ con un tiempo de adquisición de 2 segundos. Se realizó una primera tomografía 5 minutos posteriores a la inducción -tiempo que duró el posicionamiento de los animales-, en la cual se realizaron 3 cortes transversales de tórax en la zona entre el ápex cardiaco y el diafragma, a intervalos de $2 \mathrm{~mm}$ y de un grosor de $5 \mathrm{~mm}$. Posteriormente se realizaron dos tomografías a los 60 y 120 minutos posteriores a la inducción anestésica con los mismos parámetros utilizados en la primera. Éstas se realizaron con el fin de determinar las variaciones en la cantidad de tejido atelectásico a través de un procedimiento anestésico prolongado.

\section{VARIABLES A ANALIZAR}

Se realizó la medición de las distintas variables en los tres cortes en cada tiempo y se promediaron los resultados obteniendo un solo valor para cada variable.

Área Pulmonar: Para la medición de esta área pulmonar se contorneó toda el área torácica comprendida por cada pulmón exceptuando las áreas correspondientes al mediastino, corazón, diafragma y vasos importantes. La ventana utilizada fue W: $1.100 \mathrm{UH}$; L: -450 UH y en la cual se consideraron como área pulmonar todos los valores entre +100 y $-1.000 \mathrm{HU}$ (figura 1 ).

Área Atelectásica: Las áreas con valores de atenuación entre $-100 y^{+} 100 \mathrm{HU}$ encontradas en cada corte transversal fueron contorneadas utilizando un recurso del tomógrafo denominado región de interés, siendo su atenuación promedio expresada en $\mathrm{HU}$ y entregando el área en $\mathrm{mm}^{2}$ (figura 2). Para este propósito se utilizó una ventana de W: $200 \mathrm{HU}$; L: 0 HU.

Porcentaje de Atelectasia: Se calculó en base a los resultados de área pulmonar atelectásica en relación al área pulmonar de ambos pulmones por separado y al área pulmonar total.

\section{ANÁLISIS ESTADÍSTICO}

Los resultados de los tres cortes tomográficos de cada sesión fueron promediados e ingresados a una planilla MS EXCEL $®$ y se analizaron mediante el uso de estadística descriptiva en base a media aritmética. Se realizó una prueba de normalidad utilizando la prueba de Shapiro-Wilk y para determinar si existieron diferencias significativas entre las mediciones entre grupos se realizó una prueba para muestras independientes no paramétricas Test de Kruskal-Wallis y para determinar las diferencias entre tiempos se efectuó un Test exacto de Friedman. Ambos test con un nivel de significancia de $\mathrm{P}<0,05$.

El programa computacional utilizado fue el Statistix versión 8.0 para Windows (Statistix 8, Copyright@ 19852003, Analytical Software, USA).

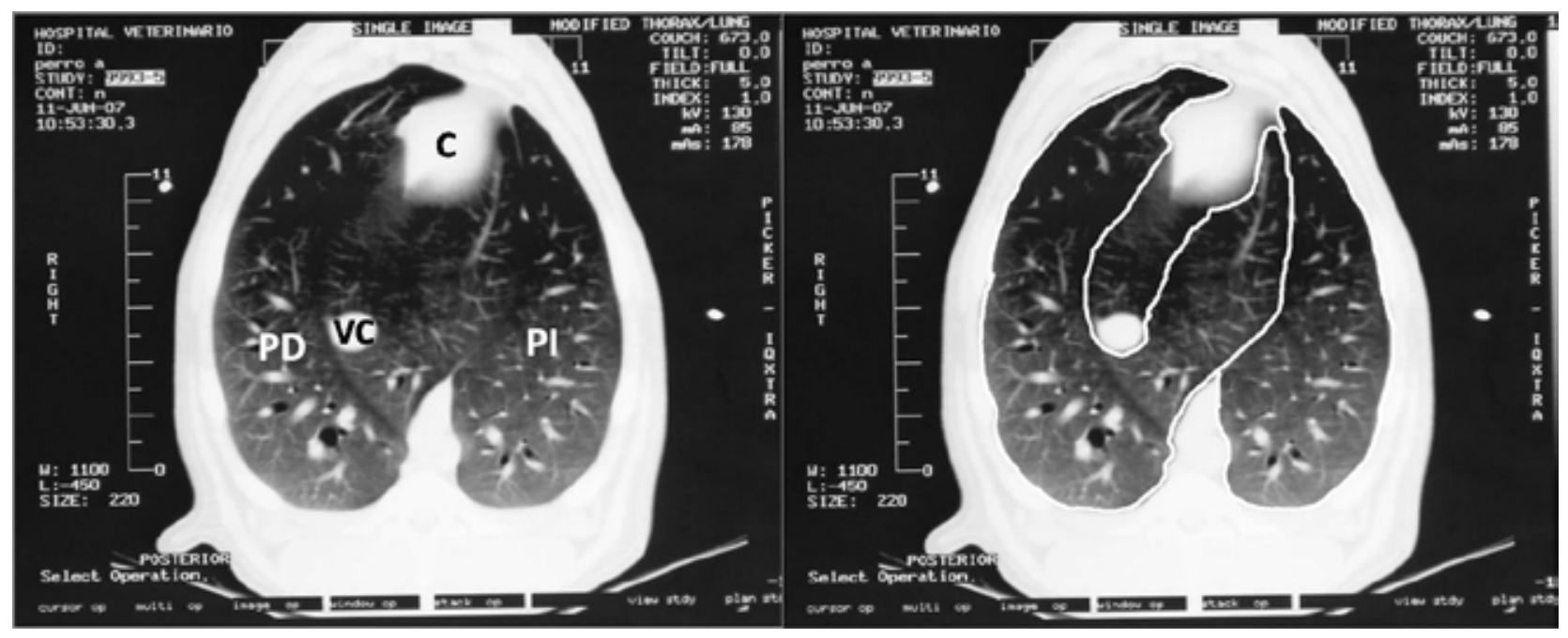

Figura 1. Imagen tomográfica torácica tomada entre el ápex del corazón y el diafragma (izquierda). Imagen en la que se explica el sistema de medición del área pulmonar (derecha). C: corazón; PD: pulmón derecho; PI: pulmón izquierdo; VC: vena cava caudal.

Transverse CT image of the thorax taken between the apex of the heart and the diaphragm (left). CT image showing the system for measuring lung area (right). C: heart; PD: right lung; PI: left lung; VC: caudal vena cava. 


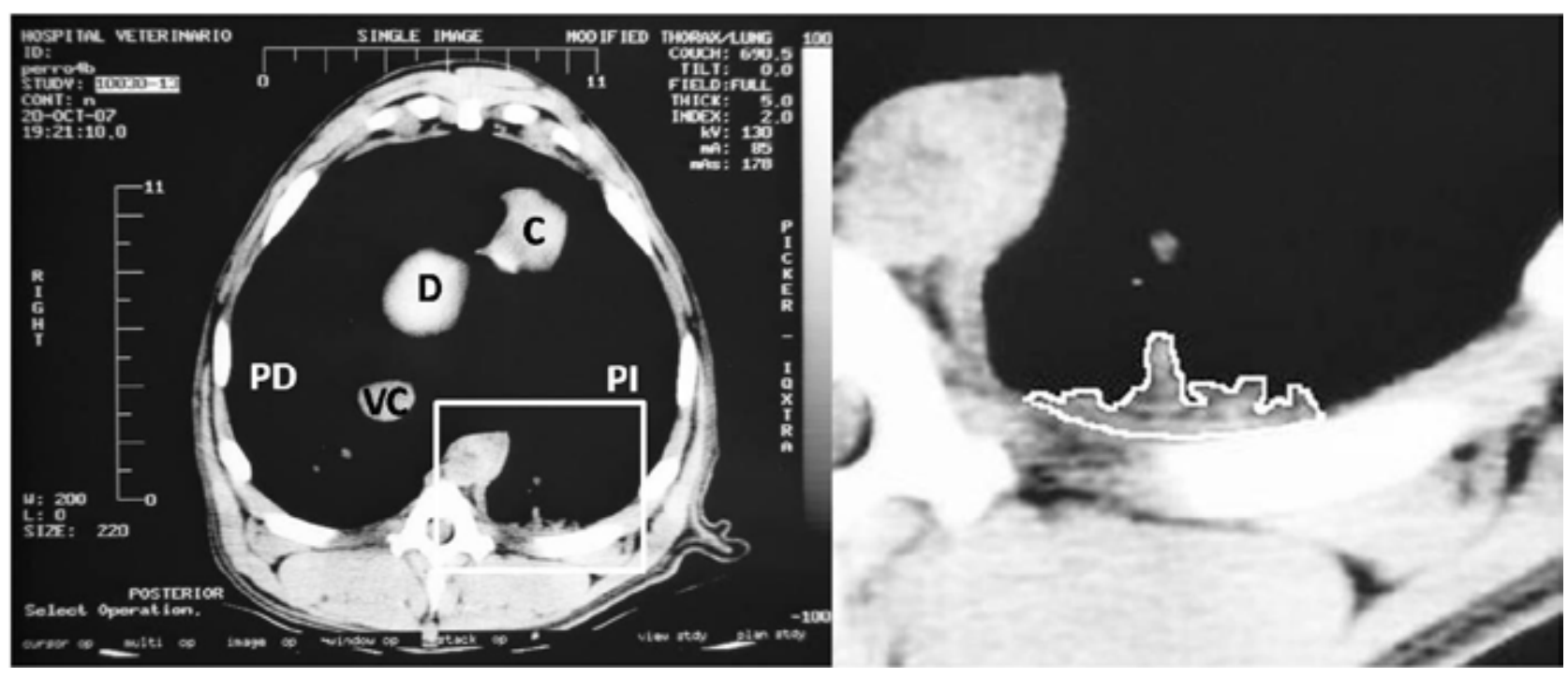

Figura 2. Imagen tomográfica torácica tomada entre el ápex del corazón y el diafragma (izquierda). Imagen en la que se explica el sistema de medición del área pulmonar atelectásica (derecha). C: corazón; D: diafragma; PD: pulmón derecho; PI: pulmón izquierdo; VC: vena cava caudal.

Transverse CT image of the thorax taken between the apex of the heart and the diaphragm (left) (left). CT image showingthe measurement system for atelectatic lung area determination (right). C: heart; D: diaphragm; PD: right lung; PI: left lung; VC: caudal vena cava.

\section{RESULTADOS}

Las imágenes obtenidas mediante TC permitieron diferenciar claramente las zonas atelectásicas y determinar por zonas de alta densidad. Además fue posible diferenciar claramente los distintos márgenes de las estructuras y los cambios en sus densidades, lo que permite realizar mediciones y comparaciones claras $\mathrm{y}$ precisas entre distintos cortes o individuos, por lo que se puede indicar a la TC como un método de gran valor diagnóstico en patologías torácicas de pequeños animales.

En el cuadro 1 se presenta el grupo sin infusión de ketamina (grupo A) y el grupo con infusión de ketamina (grupo B), indicando con una cruz a aquellos perros en los que se presentó algún grado de atelectasia $(58 \%)$ y el pulmón afectado, pudiéndose observar que un $86 \%$ de los individuos que presentaron atelectasia independientemente del grupo, ésta se presentó sólo en el pulmón izquierdo.

Las frecuencias y constantes no presentaron variaciones apreciables, a pesar de lo largo del periodo de anestesia al cual fueron sometidos los animales.

Las mediciones de las áreas pulmonares atelectásicas por perro del grupo A y del grupo B, en los cortes 1, 2 y 3 , tomadas en los minutos 0,60 y 120 posteriores a la inducción se presentan en los cuadros 2 y 3 . De la misma manera, los registros de los porcentajes de atelectasia del grupo A y del grupo B en los minutos 0 , 60 y 120 posteriores a la inducción se presentan en los cuadros 4 y 5 .
Cuadro 1. Presentación de atelectasia en pulmones derecho e izquierdo en dos grupos de perros bajo anestesia general $(n=6)$.

Presentation of atelectasis in the right and left lungs in both groups of dogs under general anesthesia $(n=6)$.

\begin{tabular}{llllll}
\hline & \multicolumn{2}{c}{ Grupo A } & & \multicolumn{2}{c}{ Grupo B } \\
\cline { 2 - 3 } \cline { 5 - 6 } Perro & D & I & & D & I \\
\hline 1 & - & + & & - & - \\
2 & + & - & & - & - \\
3 & - & - & & - & + \\
4 & - & + & & - & + \\
5 & - & + & & - & - \\
6 & - & + & & - & - \\
\hline
\end{tabular}

- No presenta atelectasia; + Presenta atelectasia; Grupo A, perros sin infusión de ketamina; Grupo B, perros con infusión de ketamina; D, pulmón derecho; I, pulmón izquierdo.

En los cuadros 2 y 3 se presentan en detalle las áreas atelectásicas observadas en los animales del grupo A y $\mathrm{B}$ respectivamente, observándose en el caso del grupo A que dos de los animales (perros 1 y 2) presentaron una mayor cantidad de tejido afectado, mientras que los otros tres presentaron cantidades menores. Del mismo modo, en el grupo B se puede observar que solamente uno de los animales (perro 3) presentó un grado mayor de tejido atelectásico, mientras que el otro animal, presentó una cantidad muy pequeña. 
Cuadro 2. Áreas atelectásicas de los pulmones derecho e izquierdo en los perros sin infusión de ketamina (grupo A), expresadas en $\mathrm{mm}^{2}$ y medidas en los minutos 0,60 y 120 posteriores a la inducción $(n=6)$.

Atelectatic areas of the right and left lungs in dogs without infusion of ketamine (group A), expressed in $\mathrm{mm}^{2}$ in minutes 0,60 and 120 post-induction $(n=6)$.

\begin{tabular}{|c|c|c|c|c|c|c|}
\hline \multirow{2}{*}{ Perro } & \multicolumn{2}{|c|}{ Minuto 0} & \multicolumn{2}{|c|}{ Minuto 60} & \multicolumn{2}{|c|}{ Minuto 120} \\
\hline & $\mathrm{D}$ & I & $\mathrm{D}$ & I & D & I \\
\hline 1 & 0,0 & 33,3 & 0,0 & 40,0 & 0,0 & 54,0 \\
\hline 2 & 58,7 & 0,0 & 68,7 & 0,0 & 80,3 & 0,0 \\
\hline 3 & 0,0 & 0,0 & 0,0 & 0,0 & 0,0 & 0,0 \\
\hline 4 & 0,0 & 0,0 & 0,0 & 3,3 & 0,0 & 10,0 \\
\hline 5 & 0,0 & 5,7 & 0,0 & 2,7 & 0,0 & 6,7 \\
\hline 6 & 0,0 & 2,3 & 0,0 & 3,0 & 0,0 & 1,3 \\
\hline
\end{tabular}

D: pulmón derecho; I: pulmón izquierdo.

En los cuadros 4 y 5 se observa el porcentaje de atelectasia pulmonar presente en los perros de ambos grupos en estudio. Fue posible determinar que existieron diferencias estadísticamente significativas entre el grupo sin infusión de ketamina y el grupo con infusión $(\mathrm{P}<0,05)$.

En cuanto al comportamiento de la atelectasia a través del tiempo, en el caso del grupo A, a pesar de observarse en algunos animales (perros 1 y 2 ) una leve tendencia al aumento en los porcentajes de atelectasia pulmonar, ésta no fue estadísticamente significativa $(\mathrm{P}>0,05)$. Por otra parte, en los animales del grupo B se aprecia una leve tendencia a la disminución, particularmente en uno de los perros (perro 3 ); sin embargo, ésta, al igual que en grupo anterior, no fue estadísticamente significativa $(P>0,05)$.

\section{DISCUSIÓN}

En este estudio el porcentaje de perros que presentaron atelectasia pulmonar fue del $58 \%$, resultado que no coincide con los presentados por el trabajo realizado por Lundquist y col (1988), quienes estudiaron la presentación de densidades pulmonares en 28 perros anestesiados con barbitúricos y donde se plantea la presentación de atelectasia en el 7\% de los perros anestesiados. Esta diferencia entre los resultados del presente estudio con los de los expuestos por Lundquist y col (1988) puede explicarse debido es que este último no administró oxígeno adicional, por lo que los animales sólo fueron ventilados con la concentración de oxígeno atmosférico; en cambio, en nuestro estudio y en el realizado por Aneli (2005) se administró una concentración inspiratoria de oxígeno de un $100 \%$, lo cual, como ya se ha explicado, disminuye el tiempo de aparición y aumenta el porcentaje de tejido pulmonar atelectásico. Otra razón que puede explicar
Cuadro 3. Áreas atelectásicas de los pulmones derecho e izquierdo en los perros con infusión de ketamina (grupo B), expresadas en $\mathrm{mm}^{2}$ y medidas en los minutos 0,60 y 120 posteriores a la inducción $(n=6)$.

Atelectatic areas of the right and left lungs in dogs with infusion of ketamine (group B), expressed in $\mathrm{mm}^{2}$ in minutes 0,60 and 120 post-induction $(n=6)$.

\begin{tabular}{|c|c|c|c|c|c|c|}
\hline \multirow{2}{*}{ Perro } & \multicolumn{2}{|c|}{ Minuto 0} & \multicolumn{2}{|c|}{ Minuto 60} & \multicolumn{2}{|c|}{ Minuto 120} \\
\hline & $\mathrm{D}$ & I & $\mathrm{D}$ & I & $\mathrm{D}$ & I \\
\hline 1 & 0,0 & 0,0 & 0,0 & 0,0 & 0,0 & 0,0 \\
\hline 2 & 0,0 & 0,0 & 0,0 & 0,0 & 0,0 & 0,0 \\
\hline 3 & 0,0 & 21,0 & 0,0 & 20,3 & 0,0 & 1,7 \\
\hline 4 & 0,0 & 0,7 & 0,0 & 4,3 & 0,0 & 3,3 \\
\hline 5 & 0,0 & 0,0 & 0,0 & 0,0 & 0,0 & 0,0 \\
\hline 6 & 0,0 & 0,0 & 0,0 & 0,0 & 0,0 & 0,0 \\
\hline
\end{tabular}

D, pulmón derecho; I, pulmón izquierdo.

Cuadro 4. Porcentaje de atelectasia pulmonar en relación al área pulmonar total, presentada en los perros sin infusión de ketamina (grupo A), en los minutos 0,60 y 120 posteriores a la inducción $(n=6)$.

Percentage of lung atelectasis in relation to the total lung area in dogs without ketamine infusion (group A), in minutes 0,60 and 120 post-induction $(n=6)$.

\begin{tabular}{cccc}
\hline Perro & Minuto 0 & Minuto 60 & Minuto 120 \\
\hline 1 & $0,26 \%$ & $0,31 \%$ & $0,44 \%$ \\
2 & $0,37 \%$ & $0,44 \%$ & $0,52 \%$ \\
3 & $0,00 \%$ & $0,00 \%$ & $0,00 \%$ \\
4 & $0,00 \%$ & $0,00 \%$ & $0,09 \%$ \\
5 & $0,04 \%$ & $0,02 \%$ & $0,05 \%$ \\
6 & $0,02 \%$ & $0,02 \%$ & $0,01 \%$ \\
\hline
\end{tabular}

Cuadro 5. Porcentaje de atelectasia pulmonar en relación al área pulmonar total, presentada en los perros con infusión de ketamina (grupo B), en los minutos 0, 60 y 120 posteriores a la inducción $(n=6)$.

Percentage of lung atelectasis in relation to the total lung area in dogs without ketamine infusion (group B), in minutes 0,60 and 120 post-induction $(n=6)$.

\begin{tabular}{cccc}
\hline Perro & Minuto 0 & Minuto 60 & Minuto 120 \\
\hline 1 & $0,00 \%$ & $0,00 \%$ & $0,00 \%$ \\
2 & $0,00 \%$ & $0,00 \%$ & $0,00 \%$ \\
3 & $0,18 \%$ & $0,17 \%$ & $0,02 \%$ \\
4 & $0,01 \%$ & $0,04 \%$ & $0,03 \%$ \\
5 & $0,00 \%$ & $0,00 \%$ & $0,00 \%$ \\
6 & $0,00 \%$ & $0,00 \%$ & $0,00 \%$ \\
\hline
\end{tabular}


los distintos resultados entre los estudios es la diferencia de pesos entre los perros en estudio, ya que Lundquist y col (1988) utilizaron perros mestizos con un promedio de peso de $18,7 \mathrm{~kg}$, mientras que en este estudio se utilizaron perros con un peso superior a los $25 \mathrm{~kg}$, situación que se asemeja a lo realizado por Aneli (2005), quien utilizó 16 perros adultos de raza Rottweiller, encontrando en ellos un porcentaje de presentación de atelectasia de un 43,75\%, similar a los resultados de esta investigación.

Destaca el hecho que en el $86 \%$ de los perros, en los que se presentó algún grado de atelectasia pulmonar, ésta se ubicó en el pulmón izquierdo, hecho que no aparece mencionado en la literatura y que pudiese deberse a un factor anatómico particular de esta especie, como lo pudiese ser la marcada diferencia en la lobulación y que predisponga a la mayor ocurrencia de colapso en ese pulmón.

Es posible además encontrar diferencias con estudios realizados en pacientes humanos, como los realizados por Rothen y col (1999), Sargent y col (1999), Hedenstierna y col (2000), Benôit y col (2002), los cuales registraron que entre un $85 \%$ y $90 \%$ de ellos presentaban atelectasia pulmonar. Según Aneli (2005), algún factor fisiológico relevante no determinado aún puede estar relacionado con la diferencia notable entre las especies. Nyman y col (1990) realizaron un estudio del deterioro del intercambio gaseoso en caballos, y Hedenstierna y col (1989) trabajaron en un modelo ovino en el cual se pudo apreciar la presencia de opacidades en los pulmones que al ser estudiadas histológicamente se determinó que eran zonas atelectásicas, acompañadas de edema y una leve congestión vascular. Ambos estudios determinaron que en las distintas especies animales se presentan opacidades pulmonares dependientes de la gravedad, pero con algunas diferencias histológicas.

El área pulmonar atelectásica varió entre 0,7 y $80,3 \mathrm{~mm}^{2}$, similar a lo presentado por el trabajo de Rothen y col (1996), quienes obtuvieron áreas con atelectasia que variaron entre 2 y $80 \mathrm{~mm}^{2}$. Ellos estudiaron el volumen de atelectasia presentado por sus pacientes con una distinta concentración inspiratoria de oxígeno. Distintos autores, entre los que destacan Rothen y col (1995), Reber y col (1996) y Edmark y col (2003), plantean que una concentración elevada de oxígeno promueve la formación de atelectasia. En el presente estudio los resultados obtenidos demostraron una pequeña cantidad de ésta, lo que hace concordar con lo planteado por Aneli (2005), quien sugiere que no está contraindicado el uso de una elevada concentración de oxígeno en la especie canina. Por otro lado, Staffieri y col (2007) describen que la diferencia de presentación de atelectasia dada por los cambios en la concentración de oxígeno, sí existe en caninos anestesiados y sometidos a una cirugía abdominal, específicamente a una ovariohisterectomía, obteniendo como resultados mayor cantidad de tejido atelectásico y una diferencia marcada entre concentraciones de $100 \%$ $\left(12,8 \pm 3,7 \mathrm{~cm}^{2}\right)$ y $40 \%\left(2,5 \pm 0,9 \mathrm{~cm}^{2}\right)$ de oxígeno, lo cual hace pensar que un factor principal en cuanto a la cantidad de tejido atelectásico presente durante la anestesia, más que la anestesia misma, es la incapacidad del diafragma de mantener la diferencia de presión durante la cirugía abdominal.

Los porcentajes totales de atelectasia pulmonar variaron entre un $0,01 \%$ y $0,52 \%$, similares a los resultados presentado por Aneli (2005), los que variaron entre 0,05\% y $1,20 \%$ y Lundquist y col (1988) en el cual el porcentaje fue de un $1 \%$. Por otra parte es distinto a los resultados del trabajo de Bletz y col (2004), el que fue realizado con 8 cerdos y en el cual se registraron las diferencias de mediciones de atelectasia entre la tomografía helicoidal y de corte dinámico donde obtuvieron resultados de $19,0 \%$ y $28,4 \%$, respectivamente. También difieren con los resultados presentados por Hedenstierna y col (1989), cuyos autores encontraron porcentajes entre un $3 \%$ y $4 \%$; estudio realizado en humanos.

El grupo tratado con ketamina presentó diferencias estadísticamente significativas con respecto al grupo sin ella. Esto concuerda con los resultados descritos por Tokics y col (1987), según los cuales sólo hubo presencia de atelectasia en uno de ocho pacientes durante la anestesia con ketamina, concluyendo que al no producirse la relajación de la pared diafragmática, así como tampoco una disminución significativa de la presión vascular pulmonar, esto impediría que disminuya de manera importante la CRF, permaneciendo ésta mayor a la capacidad de cierre, evitando así el colapso y la presentación de atelectasia. La diferencia presentada entre los estudios puede deberse a que Tokics y colaboradores realizaron su estudio con humanos, quienes, como se ha planteado anteriormente, presentan una mayor cantidad de tejido atelectásico en comparación a los perros. Además, la ketamina fue administrada en bolo como anestésico principal, lo cual difiere al presente estudio donde se administró como infusión a una dosis de $10 \mu \mathrm{g} / \mathrm{kg} / \mathrm{min}$, lo cual pudo resultar insuficiente para causar el efecto de mantención del tono muscular pero que tiene un efecto en la presión vascular pulmonar, lo cual pudiese producir una disminución en la susceptibilidad a producir atelectasia.

En relación al tiempo de aparición de atelectasia, ésta se presentó en la primera tomografía, la que se denomina en el presente estudio como la tomada en el minuto 0 , la cual en realidad era tomada aproximadamente 5 minutos luego de la inducción, tiempo que se ocupaba para el posicionamiento del animal en el tomógrafo. Esto es similar a lo expuesto por Brismar y col (1985) y Rothen y col (1995), a quienes utilizando una concentración de oxígeno de un $100 \%$, que fue la misma utilizada en el presente estudio, la atelectasia se presentó de igual manera a los 5 minutos posteriores a la inducción. Asimismo difiere de lo presentado por Rothen y col (1995) que al utilizar una concentración de oxígeno de un $40 \%$ la atelectasia se presentó a los 40 minutos luego de la inducción, lo cual se explica por lo planteado anteriormente. 
En cuanto a la influencia del tiempo de anestesia sobre la atelectasia, no hubo diferencias estadísticamente significativas entre los minutos 0,60 y 120 postinducción. Esto es explicado por Duggan y Kavanagh (2005), quienes describen el impacto del tiempo sobre la atelectasia. Ellos plantean que la máxima disminución del CRF se produce unos pocos minutos luego de la inducción anestésica y que éste no está influenciado por la profundidad ni el tiempo de anestesia.

Es de esta manera que podemos concluir que fue posible determinar que un 58\% del total de perros en estudio presentó algún grado de atelectasia pulmonar. La infusión de ketamina influyó significativamente en la presentación de atelectasia pulmonar, debido a que no hubo diferencias estadísticamente significativas en los porcentajes de atelectasia presentados entre el grupo de perros con infusión y el grupo sin infusión. No existió diferencia estadísticamente significativa entre los porcentajes de atelectasia presentados en ambos grupos a los minutos 0 , 60 y 120 posteriores a la inducción, y por último que en el $86 \%$ de los perros que presentaron atelectasia ésta se presentó en el pulmón izquierdo, sugiriendo que existe algún factor posiblemente anatómico que predispone a la mayor presentación en ese pulmón.

\section{RESUMEN}

El objetivo del estudio fue evaluar, a través de tomografía computarizada, el efecto de una infusión endovenosa de ketamina sobre la atelectasia pulmonar en perros sometidos a anestesia inhalatoria. Para esto se utilizaron 12 perros separados en dos grupos (A y B) de 6 perros cada uno. Ambos fueron sometidos al mismo protocolo anestésico que consistió en premedicación con xilacina, inducción con propofol y mantención con anestesia inhalatoria por un periodo de dos horas. Al grupo B se le administró además una infusión de ketamina. Se realizaron cortes tomográficos $1 \mathrm{~cm}$ craneal al diafragma, en los minutos 0,60 y 120 posteriores a la inducción, con el fin de monitorear la evolución en el caso de presentarse atelectasia. La totalidad del estudio fue realizado en las dependencias del Hospital Clínico Veterinario de la Universidad Austral de Chile.

Se determinó la presencia de atelectasia en diferentes grados en el $58 \%$ de los perros, observándose en la totalidad de los animales en sólo uno de los pulmones. Además se presentó en 5 perros en el grupo sin infusión de ketamina y sólo 2 en el grupo con infusión. Asimismo, las áreas atelectásicas variaron entre 0,13 y $8,03 \mathrm{~cm}^{2}$ en el grupo A y 0,07 y $2,10 \mathrm{~cm}^{2}$ en el grupo B.

La infusión de ketamina no influyó en la presentación de atelectasia pulmonar. Con respecto a la evolución de la atelectasia a través del tiempo, se apreciaron leves cambios en ambos grupos, los cuales no fueron estadísticamente significativos $(\mathrm{P}>0,05)$.

\section{REFERENCIAS}

Aneli E. 2005. Avaliaçao dos efeitos do posicionamento relacionados à opacificaçao pulmonar gravitacional dependente em caes (Canis familiaris) através da tomografía computadorizada. Memoria de título, Facultad de Medicina Veterinaria y Zootecnia de la Universidad de Sao Paulo, Sao Paulo, Brasil.

Benôit Z, S Wicky, J Fisher, P Frascarolo, C Chapuis, D Spahn, L Magnusson. 2002. The effect of increased Fio2 before tracheal extubation on postoperative atelectasis. Anesth Analg 95, 1777-1781.
Bletz C, K Markstaller, J Karmrodt, A Herweling, M Melvan, R Goetz, A Stepniak, B Eberle, H Kauczor, C Heussel, M Thelen. 2004. Quantification of atelectases in artificial respiration: spiral-CT versus dynamic single-slice CT. Rofo 176, 409-416.

Brett A, G Christensen, D Low, J Reinhardt. 2005. Computed Tomography Studies of Lung Mechanics. Proc Am Thorac Soc 2, 517-521.

Brismar B, G Hedenstierna, H Lundquist, A Strandberg, L Svensson, L Tokics. 1985. Pulmonary densities during anesthesia with muscular relaxation: a proposal of atelectasis. Anesthesiology 62, 422-428.

Brooks-Brunn J. 1995. Postoperative atelectasis and pneumonia. Heart Lung 24, 94-115.

David M, J Karmrodt, C Bletz, S David, A Herweling, H Kauczor, K Markstaller. 2005. Analysis of atelectasis, ventilated, and hyperinflated lung during mechanical by dinamic CT. Chest 128 , 3757-3770.

Don H, M Wahba, L Cuadrado, K Kelkor. 1970. The effects of anesthesia and 100 per cent oxygen on the functional residual capacity of the lungs. Anesthesiology 32, 521-529.

Duggan M, B Kavanagh. 2005. Pulmonary Atelectasis A Pathogenic Perioperative Entity. Anesthesiology 102, 838-854.

Edmark L, K Kostova-Aherdan, M Enlund, G Hedenstierna. 2003. Optimal oxygen concentration during induction of general anesthesia. Anesthesiology 98, 28-33.

Gooding J, A Dimick, M Tavakoli, G Corssen. 1977. A physiologic analysis of cardiopulmonary responses to ketamine anesthesia in non-cardiac patients. Anesth Analg 56, 813-816.

Gunnarsson L, L Tokics, H Gustavsson, G Hedenstierna. 1991. Influence of age on atelectasis formation and gas exchange impairment during general anesthesia. Br J Anaesth 66, 423-432.

Haas D, D Harper. 1992. Ketamine: A review of its pharmacologic properties and use in ambulatory anesthesia. Anesth Prog 39, 61-68.

Hedenstierna G, J Santesson. 1976. Breathing mechanics, dead space and gas exchange in the extremely obese, breathing spontaneously and during anaesthesia with intermittent positive pressure ventilation. Acta Anaesthesiol Scand 20, 248-254.

Hedenstierna G, L Tokics, A Strandberg, H Lundquist, B Brismar. 1986. Correlation of gas exchange impairment to development of atelectasis during anaesthesia and muscle paralysis. Acta Anaesthesiol Scand 30, 183-191.

Hedenstierna G, H Lundquist, B Lundh, L Tokics, A Strandberg, B Brismar, C Frostell. 1989. Pulmonary densities during anaesthesia. An Experimental study on lung morphology and gas exchange. Eur Respir J 2, 528-535.

Hedenstierna G, L Tokics, B Lund. 2000. Atelectasis formation during anesthesia: causes and measures to prevent it. J Clin Monit Comput 16, 329-335.

Hedenstierna G. 2003. Alveolar collapse and closure of airways: regular effects of anaesthesia. Clin Physiol Funct Imaging 23, 123-129.

Ko J, S Fox, R Mandsager. 2001. Anesthetic effects of ketamine or isoflurane induction prior to isoflurane anesthesia in medetomidinepremedicated dogs. J Am Anim Hosp Assoc 37, 411-419.

Lundquist H, G Hedenstierna, H Ringertz. 1988. Barbiturate anaesthesia does not cause pulmonary densities in dogs: a study using computerized axial tomography. Acta Anaesthesiol Scan 36, 626-632.

Magnusson L, D Spahn. 2003. New concepts of atelectasis during general anaesthesia. Br J Anaesth 91, 61-72.

Muir W, A Wiese, P March. 2003. Effects of morphine, lidocaine, ketamine, and morphine-lidocaine-ketamine drug combination on minimum alveolar concentration in dogs anesthetized with isoflurane. Am $J$ Vet Res 64, 1155-1160.

Nyman S, B Funkquist, C Kvart, C Frostell, L Tokics, A Strandberg, H Lundquist, B Lundh, B Brismar, G Hedenstierna. 1990. Atelectasis causes gas exchange impairment in the anaesthetised horse. Equine Vet $J$ 22, 317-324

Pedersen T, J Viby-Mogensen, C Ringsted. 1992. Anaesthetic practice and postoperative pulmonary complications. Acta Anaesthesiol Scand 36, 812-818. 
Reber A, G Engberg, B Sporre, L Kviele, H Rothen, G Wegenius, U Nylund, G Hedenstierna. 1996. Volumetric analysis of aeration in the lungs during general anaesthesia. Br J Anaesth 76, 760-766.

Robinson E. 2003. Función Respiratoria. En: Cunningham J. (ed) Fisiología Veterinaria. McGraw-Hill Interamericana, México D.F., México, Pp 468-514.

Rothen H, B Sporre, G Engberg, G Wegenius, M Högman, G Hedenstierna 1995. Influence of gas composition on recurrence of atelectasis after a reexpansion maneuver during general anesthesia. Anesthesiology 82 , 832-842.

Rothen H, B Sporre, G Engberg, G Wegenius, A Reber, G Hedenstierna. 1996. Atelectasis and pulmonary shunting during induction of general anaesthesia - can they be avoided? Acta Anaesthesiol Scand 40, 524-529.

Rothen H, P Neumann, J Berglund, J Valtysson, A Magnusson, $\mathrm{G}$ Hedenstierna. 1999. Dynamics of re-expansion of atelectasis during general anaesthesia. Br J Anaesth 82, 551-556.

Sargent M, A McEachern, D Jamieson, R Kahwaji. 1999. Atelectasis on pediatric chest CT: Comparison on sedation techniques. Pediatr Radiol 29, 509-513.

Slingsby L, A Waterman-Pearson. 2000. The post-operative analgesic effects of ketamine after canine ovariohysterectomy-a comparison between pre-or post-operative administration. Res Vet Sci 69, $147-152$
Staffieri F, D Franchini, G Carella, M Montanaro, V Valentini, B Driessen, S Grasso, A Crovace. 2007. Computed tomographic analysis of the effects of two inspired oxygen concentrations on pulmonary aeration in anesthetized and mechanically ventilated dogs. Am J Vet Res 68, 925-931.

Strandberg A, L Tokics, B Brismar, H Lundquist, G Hedenstierna. 1987. Constitutional factors promoting development of atelectasis during anaesthesia. Acta Anaesthesiol Scand 31, 21-24.

Sumano H, L Ocampo. 1997. Farmacología Veterinaria. McGraw-Hill. México D.F., México, Pp 349-514.

Tokics L, A Strandberg, B Brismar, H Lundquist, G Heenstierna. 1987. Computerized tomography of the chest and gas exchange measurements during ketamine anaesthesia. Acta Anaesthesiol Scand 66, 157-167.

Tusman G, S Bohm, F Melkun, D Staltari, C Quinzio, C Nador, E Turchetto. 2002. Alveolar recruitment strategy increases arterial oxygenation during one-lung ventilation. Ann Thorac Surg 73, 1204-1209.

Tweed W, M Minuck, D Mymin. 1972. Circulatory responses to ketamine anesthesia. Anesthesiology 37, 613-619.

Wagner A, J Walton, P Heller, J Gaynor, K Mama. 2002. Use of low doses of ketamina administered by constant rate infusion as an adjunct for postoperative analgesia in dogs. J Am Vet Med Assoc 221, 72-75.

Warner D, M Warner, E Ritman. 1996. Atelectasis and chest wall shape during halothane anesthesia. Anesthesiology 85, 49-59. 